\title{
Weighted Estimates for Bilinear Operators
}

\author{
Hua Zhu' ${ }^{1}$ and Heping Liu' \\ ${ }^{1}$ Beijing International Studies University, Beijing 100024, China \\ ${ }^{2}$ LMAM, School of Mathematical Science, Peking University, Beijing 100871, China
}

Correspondence should be addressed to Hua Zhu; zhuhua@pku.edu.cn

Received 31 May 2013; Accepted 26 November 2013; Published 6 February 2014

Academic Editor: Dashan Fan

Copyright (C) $2014 \mathrm{H}$. Zhu and H. Liu. This is an open access article distributed under the Creative Commons Attribution License, which permits unrestricted use, distribution, and reproduction in any medium, provided the original work is properly cited.

We study the boundedness of weighted multilinear operators given by products of finite vectors of Calderón-Zygmund operators. We also investigate weighted estimates for bilinear operators related to Schrödinger operator.

\section{Introduction}

Bilinear (or multilinear) operators have attracted many researchers' attention, due to their relations closely connected to the Cauchy integral along with Lipschitz curves, Calderón commutators, and compensated compactness. In [1-3] and references therein, we can see an extensive study on the Hardy space estimate of bilinear operators. In [4], we can see the bilinear operators related to a Schrödinger operator $\mathscr{L}$ and estimates of them with respect to the Hardy type space associated with the Schrödinger operator $\mathscr{L}$ under some general conditions. In our paper, we study weighted estimates for bilinear operators which have the same expression as the operators in [4].

In this paper, $N$ and $K$ will denote fixed integers $\geq 2$. Given a matrix of convolution Calderón-Zygmund kernels $\left\{K_{i}^{j}\right\}_{i=1, j=1}^{N}{ }^{K}$ on $\mathbb{R}^{d}$, we define $\left\{T_{i}^{j}\right\}_{i, j}$ as the associated CalderónZygmund operators. We denote by $L\left(f_{1}, \ldots, f_{K}\right)$ the $K$-linear operator:

$$
L\left(f_{1}, \ldots, f_{K}\right)=\sum_{i=1}^{N}\left(T_{i}^{1} f_{1}\right) \cdots\left(T_{i}^{K} f_{K}\right),
$$

which originally is defined for smooth compactly supported functions $f_{1}, \ldots, f_{K}$. For $p \leq 1, \omega \in A_{1}\left(\mathbb{R}^{d}\right)$, we denote by $H^{p}(\omega)$ the usual weighted Hardy space as defined in $[5,6]$, that is, the set of all distributions $f$ on $\mathbb{R}^{d}$ for which the maximal function $\sup _{t>0}\left|\phi_{t} * f(x)\right|$ is in $L^{p}(\omega)$, where $\phi_{t}(x)=\left(1 / t^{n}\right) \phi(x / t)$ and $\phi$ is smooth, nonzero, and compactly supported, and we also denote by $H^{p, \infty}(\omega)$ the weak $H^{p}$ as defined in [7], that is, the set of all distributions $f$ on $\mathbb{R}^{d}$ for which the maximal function $\sup _{t>0}\left|\phi_{t} * f(x)\right|$ is in weak $L^{p}(\omega)$. If $\omega(x) \equiv 1$, the weak Hardy space $H^{1, \infty}\left(\mathbb{R}^{d}\right)=H^{1, \infty}(\omega)$ was introduced by Feerman and Soria in [8]. $H^{p, \infty}\left(\mathbb{R}^{d}\right)$ first appeared in [9] (see also [10]).

Theorem 1. Assume that $p_{1}, \ldots, p_{K}>1$ are given and let $r=\left(p_{1}^{-1}+\cdots+p_{K}^{-1}\right)^{-1}$ be their harmonic mean. We also assume that the harmonic mean of any proper subset of the $p_{j}$ 's is greater than 1. If, for all $\left(f_{1}, \ldots, f_{K}\right) \in\left(C_{0}^{\infty}\right)^{K}$, the K-linear operator L satisfies

$$
\int L\left(f_{1}, \ldots, f_{K}\right) d x=0
$$

Then, for $\omega \in A_{1}\left(\mathbb{R}^{d}\right)$, one has following conclusions.

(1) If $r>1, L$ maps $L^{p_{1}}(\omega) \times \cdots \times L^{p_{K}}(\omega) \rightarrow L^{r}(\omega)$.

(2) If $1 \geq r>d /(d+1)$, L maps $L^{p_{1}}(\omega) \times \cdots \times L^{p_{K}}(\omega) \rightarrow$ $H^{r}(\omega)$.

(3) If $r=d /(d+1)$, L maps $L^{p_{1}}(\omega) \times \cdots \times L^{p_{K}}(\omega) \rightarrow$ $L^{r, \infty}(\omega)$.

Let $\mathscr{L}=-\Delta+V$ be a Schrödinger operator on $\mathbb{R}^{d}, d \geq 3$, where $V \not \equiv 0$ is a fixed nonnegative potential. We will assume 
that $V$ belongs to reverse Hölder class $\mathrm{RH}_{s}\left(\mathbb{R}^{d}\right)$ for some $s \geq$ $d / 2$; that is, there exists $C=C(s, V)>0$ such that

$$
\left(\frac{1}{|B|} \int_{B} V(x)^{s} d x\right)^{1 / s} \leq C\left(\frac{1}{|B|} \int_{B} V(x) d x\right)
$$

for every ball $B \subset \mathbb{R}^{d}$. In what follows, $B(x, r)$ denotes the ball centered at $x$ and of the radius $r$. Trivially, $\mathrm{RH}_{q}\left(\mathbb{R}^{d}\right) \subset$ $\mathrm{RH}_{p}\left(\mathbb{R}^{d}\right)$ provided $1<p \leq q<\infty$. It is well known that if $V \in \mathrm{RH}_{q}\left(\mathbb{R}^{d}\right)$ for some $q>1$, then there exists $\varepsilon>0$, which depends only on $d$ and the constant $C$ in (3), such that $V \in \mathrm{RH}_{q+\varepsilon}\left(\mathbb{R}^{d}\right)$ (see [11]). Throughout this paper, we always assume that $0 \not \equiv V \in \mathrm{RH}_{d / 2}$. Thus, $V \in \mathrm{RH}_{q_{0}}$ for some $q_{0}>$ $d / 2$.

Let $\left\{T_{t}^{\mathscr{L}}\right\}_{t>0}$ be the semigroup of linear operators generated by $\mathscr{L}$ and let $T_{t}^{\mathscr{L}}(x, y)$ be their kernels; that is,

$$
\begin{aligned}
T_{t}^{\mathscr{L}} f(x) & =e^{-t \mathscr{L}} f(x) \\
& =\int_{\mathbb{R}^{d}} T_{t}^{\mathscr{L}}(x, y) f(y) d y, \\
& \text { for } t>0, f \in L^{2}\left(\mathbb{R}^{d}\right) .
\end{aligned}
$$

By the Trotter product formula (cf. [12]),

$$
0 \leq T_{t}^{\mathscr{L}}(x, y) \leq H_{t}(x, y)=(4 \pi t)^{-d / 2} \exp \left(-\frac{|x-y|^{2}}{4 t}\right)
$$

The maximal function with respect to the semigroup $\left\{T_{t}^{\mathscr{L}}\right\}_{t>0}$ is defined by

$$
\mathscr{T}^{*} f(x)=\sup _{t>0}\left|T_{t}^{\mathscr{L}} f(x)\right| .
$$

The weighted Hardy-type space related to $\mathscr{L}$ is naturally defined by (see [13])

$$
\begin{array}{r}
H_{\mathscr{L}}^{1}(\omega)=\left\{f \in L^{1}(\omega): \mathscr{T}^{*} f(x) \in L^{1}(\omega)\right\}, \\
\text { with }\|f\|_{H_{\mathscr{L}}^{1}(\omega)}=\left\|\mathscr{T}^{*} f\right\|_{L^{1}(\omega)} .
\end{array}
$$

Following [14], we define the auxiliary function $\rho(x, V)=$ $\rho(x)$ by

$$
\rho(x)=\rho(x, V)=\sup \left\{r>0: \frac{1}{r^{d-2}} \int_{B(x, r)} V(y) d y \leq 1\right\} .
$$

The auxiliary function $\rho(x)$ plays an important role in studying the boundedness of singular integral operators related to the Schrödinger operator $\mathscr{L}$ as well as the atomic decomposition of $H_{\mathscr{L}}^{1}$ and $H_{\mathscr{L}}^{1}(\omega)$ (see [13-15]).

In our paper, we also consider the following bilinear operators:

$$
T^{ \pm}(f, g)(x)=\left(T_{1} f\right)(x)\left(T_{2} g\right)(x) \pm\left(T_{2} f\right)(x)\left(T_{1} g\right)(x),
$$

where $f \in L^{p}(\omega), g \in L^{q}(\omega)$ with $1<p, q<\infty$ and $1 / p+1 / q=1, T_{i}(i=1,2)$ are Calderón-Zygmund operators related to $\mathscr{L}$ and satisfy the following two conditions. (i) There exist parallel Calderón-Zygmund operators $\widetilde{T}_{i}$ related to the Laplacian $\Delta$ and a constant $\delta>0$ such that

$$
\left|T_{i}(x, y)-\widetilde{T}_{i}(x, y)\right| \leq \frac{C}{\rho(y)^{\delta}|x-y|^{d-\delta}}, \quad x \neq y,
$$

where $T_{i}(x, y)$ and $\widetilde{T}_{i}(x, y)$ denote the kernels of $T_{i}$ and $\widetilde{T}_{i}$, respectively.

(ii) One of the parallel bilinear operators

$$
\begin{aligned}
\widetilde{T}^{ \pm}(f, g)(x)= & \left(\widetilde{T}_{1} f\right)(x)\left(\widetilde{T}_{2} g\right)(x) \\
& \pm\left(\widetilde{T}_{2} f\right)(x)\left(\widetilde{T}_{1} g\right)(x)
\end{aligned}
$$

has the vanishing moment; that is, either $\widetilde{T}^{+}$or $\widetilde{T}^{-}$ satisfies

$$
\int_{\mathbb{R}^{d}} \widetilde{T}^{ \pm}(f, g)(x) d x=0 \quad \forall f, g \in C_{c}^{\infty}\left(\mathbb{R}^{d}\right) .
$$

We will show that either $T^{+}$or $T^{-}$is bounded from $L^{p}(\omega) \times$ $L^{q}(\omega)$ to $H_{\mathscr{L}}^{1}(\omega)$.

Theorem 2. Suppose that the bilinear operators $T^{ \pm}$are defined as above. Let $1<p, q<\infty$ and $1 / p+1 / q=1$. Then either $T^{+}$ or $T^{-}$(but not both), which corresponds to the parallel bilinear operator satisfying (12), maps $L^{p}(\omega) \times L^{q}(\omega)$ into $H_{\mathscr{L}}^{1}(\omega)$ and there exists a constant $C>0$ such that

$$
\left\|T^{ \pm}(f, g)\right\|_{H_{\mathscr{L}}^{1}(\omega)} \leq C\|f\|_{L^{p}(\omega)}\|g\|_{L^{q}(\omega)} .
$$

This paper is organized as follows. In Section 2, we give some notation and preliminary estimates on $\rho(x)$ and the kernel $T_{t}(x, y)$ which have been proved in [14-16]. In Section 3, we prove Theorem 1, and in Section 4 we prove Theorem 2.

Throughout this paper, we will use $C$ to denote a positive constant, which is not necessarily the same at each occurrence. By $A \sim B$, we mean that there exists a constant $C>1$, such that $C^{-1} \leq A / B \leq C$. For a given ball $B$, we denote by $B^{*}$ the concentric ball with twice radius, and $B^{* *}=\left(B^{*}\right)^{*}$.

\section{Preliminaries}

Throughout this paper, we will denote $\omega(E):=\int_{E} \omega(x) d x$ for any set $E \subset \mathbb{R}^{d}$. For $1 \leq p \leq \infty$, denote by $p^{\prime}$ the adjoint number of $p$; that is, $1 / p+1 / p^{\prime}=1$.

We review some needed background about Muckenhoupt weights. A weight $\omega$ is a nonnegative locally integrable function on $\mathbb{R}^{d}$. We say that $\omega \in A_{p}\left(\mathbb{R}^{d}\right), 1<p<\infty$, if there exists a constant $C$ such that

$$
\left(\frac{1}{|B|} \int_{B} \omega\right)\left(\frac{1}{|B|} \int_{B} \omega^{1-p^{\prime}}\right)^{p-1} \leq C,
$$

for every ball $B \subset \mathbb{R}^{d}$. The class $A_{1}\left(\mathbb{R}^{d}\right)$ is defined replacing the above inequality by

$$
M \omega(x) \leq C \omega(x) \quad \text { a.e. } x \in \mathbb{R}^{d},
$$

where $M$ denotes the standard Hardy-Littlewood operator. 
Let $0<p<\infty$ and let $\omega$ be a locally integrable nonnegative function. We denote the weighted space $L^{p}\left(\mathbb{R}^{d}, \omega(x) d x\right)$ by $L^{p}(\omega)$ and set

$$
\|f\|_{L^{p}(\omega)}=\left(\int_{\mathbb{R}^{d}}|f(x)|^{p} \omega(x) d x\right)^{1 / p} .
$$

We also denote the weak $L^{p}(\omega)$ by $L^{p, \infty}(\omega)$ and set

$$
\|f\|_{L^{p, \infty}(\omega)}=\sup _{\lambda>0} \lambda\left[\omega\left(\left\{x \in \mathbb{R}^{d}:|f(x)|>\lambda\right\}\right)\right]^{1 / p} .
$$

We first sum up some properties of weights in the following results.

Lemma 3. One has the following properties.

(i) $A_{p} \subset A_{q}$ for $1 \leq p \leq q<\infty$.

(ii) Let $p \in(1, \infty)$. Then $\omega \in A_{p}$ if and only if $\omega^{1-p^{\prime}} \in$ $A_{p^{\prime}}$.

(iii) If $\omega \in A_{p}, 1 \leq p<\infty$, then there exists $\varepsilon>0$ such that $\omega^{1+\varepsilon} \in A_{p}$.

(iv) If $\omega \in A_{p}, 1 \leq p<\infty$, then the measure $\omega(x) d x$ is doubling: precisely, for all $\lambda>1$ and all cubes $Q$ one has

$$
\omega(\lambda Q) \leq \lambda^{d p}[\omega]_{A_{p}} \omega(Q),
$$

(where $[\omega]_{A_{p}}$ denotes the $A_{p}$ Muckenhoupt characteristic constant and $\lambda Q$ denotes the cube with the same center as $Q$ and side length $\lambda$ times the side length of Q).

(v) $A_{\infty}\left(\mathbb{R}^{d}\right)=\bigcup_{p \geq 1} A_{p}\left(\mathbb{R}^{d}\right)$.

Proof. Properties are standard; see, for instance, [17-19].

Lemma 4. Let $B$ be a ball of $\mathbb{R}^{d}$ and let $E$ be any measurable subset of $B$. Let $\omega \in A_{p}, 1 \leq p<\infty$. Then, there exist constants $C_{1}>0, C_{2}>0$, and $\delta>0$ such that

$$
C_{1}\left(\frac{|E|}{|B|}\right)^{p} \leq \frac{\omega(E)}{\omega(B)} \leq C_{2}\left(\frac{|E|}{|B|}\right)^{\delta} .
$$

Proof. For the proof, we refer to [17].

Next we recall some basic properties of the auxiliary function $\rho(x)$. It is known that $0<\rho(x)<\infty$ for any $x \in \mathbb{R}^{d}$ (cf. [14]). Therefore,

$$
\mathbb{R}^{d}=\bigcup_{k=-\infty}^{\infty} \Omega_{k}
$$

where

$$
\Omega_{k}=\left\{x \in \mathbb{R}^{d}: 2^{-k-1} \leq \rho(x)<2^{-k}\right\} .
$$

Lemma 5 (see [15]). There exist a constant $N=N(V)$ and a sequence of points $\left\{x_{(k, \alpha)} \in \Omega_{k}: k, \alpha \in \mathbb{Z}\right\}$ such that the family of critical balls $\left\{B_{(k, \alpha)}\right\}$, defined by $B_{(k, \alpha)}=\left\{x \in \mathbb{R}^{d}\right.$ : $\left.\left|x-x_{(k, \alpha)}\right|<\rho\left(x_{(k, \alpha)}\right)\right\}$, satisfies (i) $\bigcup_{(k, \alpha)} B_{(k, \alpha)}=\mathbb{R}^{d}$,

(ii) $\#\left\{\left(k^{\prime}, \alpha^{\prime}\right): B_{\left(k^{\prime}, \alpha^{\prime}\right)}^{* *} \cap B_{(k, \alpha)}^{* *} \neq \emptyset\right\} \leq N$ for every $(k, \alpha)$.

Furthermore, there exists a family of $C^{\infty}$ functions $\left\{\psi_{(k, \alpha)}\right\}_{k, \alpha \in \mathbb{Z}}$ such that

(iii) $\operatorname{supp} \psi_{(k, \alpha)} \subseteq B_{(k, \alpha)}^{*}$,

(iv) $0 \leq \psi_{(k, \alpha)}(x) \leq 1$ and $\sum_{(k, \alpha)} \psi_{(k, \alpha)}(x)=1$ for all $x \in$ $\mathbb{R}^{d}$

(v) $\left\|\nabla \psi_{(k, \alpha)}\right\|_{L^{\infty}} \leq C / \rho\left(x_{(k, \alpha)}\right)$.

Lemma 6 (see [14]). There exist $C>0$ and $k_{0} \geq 1$ so that, for all $x, y \in \mathbb{R}^{d}$,

$$
\begin{aligned}
& C^{-1} \rho(x)\left(1+\frac{|x-y|}{\rho(x)}\right)^{-k_{0}} \\
& \quad \leq \rho(y) \leq C \rho(x)\left(1+\frac{|x-y|}{\rho(x)}\right)^{k_{0} /\left(k_{0}+1\right)} .
\end{aligned}
$$

In particular, $\rho(x) \sim \rho(y)$ when $y \in B(x, r)$ and $r \leq C \rho(x)$.

To prove Theorem 2, we need the following estimates for the kernel $T_{t}^{\mathscr{L}}(x, y)$.

Lemma 7 (see $[15,16])$. For every $N$, there is a constant $C_{N}>$ 0 such that

$$
\begin{aligned}
0 & \leq T_{t}^{\mathscr{L}}(x, y) \\
& \leq C_{N} t^{-(d / 2)}\left(1+\frac{\sqrt{t}}{\rho(x)}+\frac{\sqrt{t}}{\rho(y)}\right)^{-N} \exp \left(-\frac{|x-y|^{2}}{5 t}\right) .
\end{aligned}
$$

Also, there exists a constant $C>0$ such that

$$
\left|T_{t}^{\mathscr{L}}(x, y)-H_{t}(x, y)\right| \leq \frac{C}{\rho(x)^{\sigma}|x-y|^{d-\sigma}},
$$

where $\sigma=2-d / q_{0}>0$.

\section{The Proof of Theorem 1}

We fix $p_{1}, \ldots, p_{K}>1$ and we let $r$ be their harmonic mean. For $r>1$, we can get the result just by Hölder's inequality and the $L^{p}(\omega)$ boundedness of Calderón-Zygmund operators. Then we just need to study the case $r \leq 1$. Fix a smooth compactly supported function $\phi$ in $\mathbb{R}^{d}$, an $x_{0} \in \mathbb{R}^{d}$, and define $\phi_{t, x_{0}}(x)=\left(1 / t^{n}\right) \phi\left(\left(x-x_{0}\right) / t\right)$. Without loss of generality we may assume that $\phi$ is supported in $|x| \leq 1$. We need to show that $\sup _{t>0}\left|\int \phi_{t, x_{0}} L\left(f_{1}, \ldots, f_{K}\right) d x\right|$ is in $L^{r}(\omega)$ when $r>n /(n+1)$ and in $L^{r, \infty}(\omega)$ when $r=n /(n+1)$. We also fix a smooth cutoff $\eta(x)$ such that $\eta \equiv 1$ on $|x|<2$ and is supported in $|x|<4$. We call for simplicity $\eta_{0}(x)=$ 
$\eta\left(\left(x_{0}-x\right) / t\right)$ and $\eta_{1}(x)=1-\eta_{0}(x)$. We now decompose $L\left(f_{1}, \ldots, f_{K}\right)=L_{0}+L_{1}+\cdots+L_{K+1}$, where

$$
\begin{aligned}
L_{0} & =L\left(\eta_{0} f_{1}, \eta_{0} f_{2}, \ldots, \eta_{0} f_{K}\right), \\
L_{1} & =\sum_{j=1}^{K} L\left(f_{1}, \ldots, \eta_{1} f_{j}, \ldots, f_{K}\right), \\
L_{2} & =\sum_{\substack{1 \leq j, l \leq K \\
j<l}} L\left(f_{1}, \ldots, \eta_{1} f_{j}, \ldots, \eta_{1} f_{l}, \ldots, f_{K}\right), \\
& \vdots \\
L_{K+1} & =(-1)^{K} L\left(\eta_{1} f_{1}, \eta_{1} f_{2}, \ldots, \eta_{1} f_{K}\right) .
\end{aligned}
$$

In each $L_{u}$ above exactly $u$ functions among the $f_{j}$ 's are multiplied by $\eta_{1}$ and the remaining are left intact.

For any fixed $i, k$ and any $x$ such that $\left|x-x_{0}\right| \leq t$ we have

$$
\begin{aligned}
& \sup _{t>0}\left|T_{i}^{k}\left(\eta_{1} f\right)(x)-T_{i}^{k}\left(\eta_{1} f\right)\left(x_{0}\right)\right| \\
& \quad \leq \sup _{t>0}\left|\int\left(K_{i}^{k}(x-y)-K_{i}^{k}\left(x_{0}-y\right)\right) \eta_{1}(y) f(y) d y\right| \\
& \quad \leq C \sup _{t>0} \int_{\left|y-x_{0}\right| \geq t}\left|x-x_{0}\right|\left|y-x_{0}\right|^{-n-1}|f(y)| d y \\
& \quad \leq C M(|f|)\left(x_{0}\right),
\end{aligned}
$$

where $M(f)\left(x_{0}\right)$ is the Hardy-Littlewood maximal function of $f$ at the point $x_{0}$. We denote the maximal truncated operator of $T_{i}^{j}$ by $\left(T_{i}^{j}\right)^{*}$. We begin with the $L_{1}$ :

$$
\begin{aligned}
L_{1}= & \sum_{j=1}^{K} L\left(f_{1}, \ldots,\left(\eta_{1} f_{j}\right)(x)-\left(\eta_{1} f_{j}\right)\left(x_{0}\right), \ldots, f_{K}\right) \\
& +\sum_{j=1}^{K} L\left(f_{1}, \ldots,\left(\eta_{1} f_{j}\right)\left(x_{0}\right), \ldots, f_{K}\right) .
\end{aligned}
$$

Then we have

$$
\begin{aligned}
& I=\sup _{t>0}\left|\int \phi_{t, x_{0}} L_{1} d x\right| \\
& \leq \sum_{j=1}^{K} \sum_{i=1}^{N} \sup _{t>0} \int\left|\phi_{t, x_{0}}\right| \prod_{\substack{1 \leq k \leq K \\
k \neq j}}\left|T_{i}^{k} f_{k}\right| \\
& \times\left(\left|T_{i}^{j}\left(\eta_{1} f_{j}\right)(x)-T_{i}^{j}\left(\eta_{1} f_{j}\right)\left(x_{0}\right)\right|\right. \\
& \left.+\quad+\left|T_{i}^{j}\left(\eta_{1} f_{j}\right)\left(x_{0}\right)\right|\right) d x \\
& \leq C \sum_{j=1}^{K} \sum_{i=1}^{N} M\left(\prod_{\substack{1 \leq k \leq K \\
k \neq j}}\left|T_{i}^{k} f_{k}\right|\right)\left(x_{0}\right) \\
& \quad \times\left(M\left(f_{j}\right)\left(x_{0}\right)+\left(T_{i}^{j}\right)^{*} f_{j}\left(x_{0}\right)\right) .
\end{aligned}
$$

Define $\sigma_{j}$ by $\sigma_{j}^{-1}+p_{j}^{-1}=r^{-1}$. By Hölder inequality and the boundedness of $M$ and $\left(T_{i}^{j}\right)^{*}$, the $L^{r}(\omega)$ norm in $x_{0}$ of $I$ is bounded by

$$
\begin{aligned}
& C \sum_{j=1}^{K} \sum_{i=1}^{N}\left(\left\|M\left(f_{j}\right)\right\|_{L^{p_{j}}(\omega)}+\left\|\left(T_{i}^{j}\right)^{*}\left(f_{j}\right)\right\|_{L^{p_{j}}(\omega)}\right) \\
& \times\left\|M\left(\prod_{\substack{1 \leq k \leq K \\
k \neq j}}\left|T_{i}^{k} f_{k}\right|\right)\right\|_{L^{\sigma_{j}}(\omega)} \\
& \leq C \sum_{j=1}^{K} \sum_{i=1}^{N}\left\|f_{j}\right\|_{L^{p_{j}}(\omega)}\left\|\prod_{\substack{1 \leq k \leq K \\
k \neq j}}\left|T_{i}^{k} f_{k}\right|\right\|_{L^{\sigma_{j}}(\omega)} \\
& \leq C \sum_{j=1}^{K} \sum_{i=1}^{N}\left\|f_{j}\right\|_{L^{p_{j}}(\omega)} \prod_{\substack{1 \leq k \leq K \\
k \neq j}}\left\|T_{i}^{k} f_{k}\right\|_{L^{p_{k}}(\omega)} \\
& \leq C \sum_{j=1}^{K}\left\|f_{j}\right\|_{L^{p_{j}}(\omega)} \prod_{\substack{1 \leq k \leq K \\
k \neq j}}\left\|f_{k}\right\|_{L^{p_{k}}(\omega)} \\
& =C \prod_{k=1}^{K}\left\|f_{k}\right\|_{L^{p_{k}(\omega)}} .
\end{aligned}
$$

We obtain that

$$
\begin{gathered}
\left\|\sup _{t>0}\left|\int \phi_{t, x 0} L_{1} d x\right|\right\|_{L^{r}(\omega)} \leq C \prod_{k-1}^{K}\left\|f_{k}\right\|_{L^{p_{k}(\omega)}}, \\
\omega\left(\left\{x_{0}: \sup _{t>0}\left|\int \phi_{t, x_{0}} L_{1} d x\right|>\lambda\right\}\right) \leq C \lambda^{-r} \prod_{k=1}^{K}\left\|f_{k}\right\|_{L^{p_{k}(\omega)}}^{r} .
\end{gathered}
$$

For $L_{2}$, we write $L_{2}=L_{21}+L_{22}+L_{23}+L_{24}$ where

$$
\begin{aligned}
L_{21}=-\sum_{\substack{1 \leq j, l \leq K \\
j<l}} L\left(f_{1}, \ldots,\left(\eta_{1} f_{j}\right)(x)\right. & \\
& -\left(\eta_{1} f_{j}\right)\left(x_{0}\right), \ldots,\left(\eta_{1} f_{l}\right)(x) \\
& \left.-\left(\eta_{1} f_{l}\right)\left(x_{0}\right), \ldots, f_{K}\right), \\
L_{22}=-\sum_{\substack{1 \leq j, l \leq K \\
j<l}} L\left(f_{1}, \ldots,\left(\eta_{1} f_{j}\right)\left(x_{0}\right), \ldots,\left(\eta_{1} f_{l}\right)(x)\right. & \left.-\left(\eta_{1} f_{l}\right)\left(x_{0}\right), \ldots, f_{K}\right),
\end{aligned}
$$




$$
\begin{gathered}
L_{23}=-\sum_{\substack{1 \leq j, l \leq K \\
j<l}} L\left(f_{1}, \ldots,\left(\eta_{1} f_{j}\right)(x)\right. \\
\left.\quad-\left(\eta_{1} f_{j}\right)\left(x_{0}\right), \ldots,\left(\eta_{1} f_{l}\right)\left(x_{0}\right), \ldots, f_{K}\right), \\
\begin{array}{c}
L_{24}=-\sum_{\substack{1 \leq j, l \leq K \\
j<l}} L\left(f_{1}, \ldots,\left(\eta_{1} f_{j}\right)\left(x_{0}\right), \ldots,\right. \\
\left.\left(\eta_{1} f_{l}\right)\left(x_{0}\right), \ldots, f_{K}\right) .
\end{array}
\end{gathered}
$$

Using the same reason as before, we have the following estimate of $L_{2 u}, u=1,2,3,4$ :

$$
\begin{aligned}
\sup _{t>0}\left|\int \phi_{t, x_{0}} L_{2 u} d x\right| & \\
\leq & C \sum_{\substack{1 \leq j, l \leq K \\
j<l}} \sum_{i=1}^{K} M\left(\prod_{\substack{1 \leq k \leq K \\
k \neq j, l}}\left|T_{i}^{k} f_{k}\right|\right) \\
& \times\left(x_{0}\right)\left(\left(C_{j} f_{j}\right)\left(x_{0}\right)\left(C_{l} f_{l}\right)\left(x_{0}\right)\right),
\end{aligned}
$$

where each $C_{j} f_{j}$ is either $M\left(f_{j}\right)$ or $\left(T_{i}^{j}\right)^{*} f_{j}$ and therefore $\left\|C_{j} f_{j}\right\|_{L^{p_{j}}(\omega)} \leq C\left\|f_{j}\right\|_{L^{p_{j}(\omega)}}$. Define $\sigma_{j l}$ by $\sigma_{j l}^{-1}+p_{j}^{-1}+p_{l}^{-1}=r^{-1}$. For each $u=1,2,3,4$, Hölder inequality implies that

$$
\begin{aligned}
& \left\|\sup _{t>0}\left|\int \phi_{t, x_{0}} L_{2 u} d x\right|\right\|_{L^{r}(\omega)} \\
& \leq C \sum_{\substack{1 \leq j, l \leq K \\
j<l}} \sum_{i=1}^{K}\left\|M\left(\prod_{\substack{1 \leq k \leq K \\
k \neq j, l}}\left|T_{i}^{k} f_{k}\right|\right)\right\|_{L^{\sigma_{j l}(\omega)}} \\
& \quad \times\left(\left\|C_{j} f_{j}\right\|_{L^{p_{j}}(\omega)}\left\|C_{l} f_{l}\right\|_{L^{p_{l}}(\omega)}\right) \\
& \leq C \sum_{\substack{1 \leq j, l \leq K \\
j<l}} \sum_{i=1}^{K}\left\|\prod_{\substack{1 \leq k \leq K \\
k \neq j, l}}\left|T_{i}^{k} f_{k}\right|\right\|_{L^{\sigma_{j l} l}(\omega)} \\
& \quad \times\left(\left\|f_{j}\right\|_{L^{p_{j}}(\omega)}\left\|f_{l}\right\|_{L^{p_{l} l}(\omega)}\right) \\
& \leq C \sum_{\substack{1 \leq j, l \leq K \\
j<l}} \sum_{\substack{i=1 \\
j<1}} \prod_{\substack{1 \leq k \leq K \\
k \neq j, l}}\left\|T_{i}^{k} f_{k}\right\|_{L^{p_{k}(\omega)}}
\end{aligned}
$$

$$
\begin{aligned}
& \times\left(\left\|f_{j}\right\|_{L^{p_{j}}(\omega)}\left\|f_{l}\right\|_{L^{p_{l}}(\omega)}\right) \\
\leq & C \sum_{\substack{1 \leq j, l \leq K \\
j<l}} \prod_{\substack{1 \leq k \leq K \\
k \neq j, l}}\left\|f_{k}\right\|_{L^{p_{k}}(\omega)} \\
& \times\left(\left\|f_{j}\right\|_{L^{p_{j}}(\omega)}\left\|f_{l} f_{l}\right\|_{L^{p_{l}(\omega)}}\right) \\
\leq & C \prod_{1 \leq k \leq K}\left\|f_{k}\right\|_{L^{p_{k}}(\omega)} \cdot
\end{aligned}
$$

Then we get

$$
\begin{gathered}
\left\|\sup _{t>0}\left|\int \phi_{t, x 0} L_{1} d x\right|\right\|_{L^{r}(\omega)} \leq C \prod_{k-1}^{K}\left\|f_{k}\right\|_{L^{p} k(\omega)}, \\
\omega\left(\left\{x_{0}: \sup _{t>0}\left|\int \phi_{t, x_{0}} L_{2} d x\right|>\lambda\right\}\right) \leq C \lambda^{-r} \prod_{k=1}^{K}\left\|f_{k}\right\|_{L^{p_{k}(\omega)}}^{r} .
\end{gathered}
$$

For $L_{3}, L_{4}, \ldots, L_{K+1}$, by the similar method, we get the desired conclusions. Now we turn to the term $L_{0}$, and we use two inequalities which have been proved in [3] as follows:

$$
\begin{array}{r}
\sup _{t>0}\left|\int \phi_{t, x_{0}} L_{0} d x\right| \leq C \prod_{k=1}^{K}\left(M\left(\left|f_{k}\right|^{s_{k}}\right)\left(x_{0}\right)\right)^{1 / s_{k}} \\
\text { when } r>\frac{d}{(d+1)}, \\
\sup _{t>0}\left|\int \phi_{t, x_{0}} L_{0} d x\right| \leq C \prod_{k=1}^{K}\left(M\left(\left|f_{k}\right|^{p_{k}}\right)\left(x_{0}\right)\right)^{1 / p_{k}} \\
\text { when } r=\frac{d}{(d+1)},
\end{array}
$$

where $1<s_{j}<p_{j}$.

Then, for $r>d /(d+1)$, we get

$$
\begin{aligned}
& \left\|\sup _{t>0}\left|\int \phi_{t, x_{0}} L_{0} d x\right|\right\|_{L^{r}(\omega)} \\
& \leq C\left\|\prod_{k=1}^{K}\left(M\left(\left|f_{k}\right|^{s_{k}}\right)\left(x_{0}\right)\right)^{1 / s_{k}}\right\|_{L^{r}(\omega)} \\
& \leq C \prod_{k=1}^{K}\left\|\left(M\left(\left|f_{k}\right|^{s_{k}}\right)\left(x_{0}\right)\right)^{1 / s_{k}}\right\|_{L^{p_{k}}(\omega)} \\
& \leq C \prod_{k=1}^{K}\left\|f_{k}\right\|_{L^{p_{k}}(\omega)},
\end{aligned}
$$

where we use $p_{k} / s_{k}>1$, and we obtain conclusion (2) of Theorem 1. 
For conclusion (3), let $\varepsilon_{0}=\lambda / C, \varepsilon_{K}=1$, and $\varepsilon_{1}, \ldots, \varepsilon_{K-1}>$ 0 be arbitrary; then, by using $(36)$ and the weak $(1,1)$ result for the Hardy-Littlewood maximal function, we can get

$$
\begin{aligned}
& \omega\left(\left\{x_{0}: \sup _{t>0}\left|\int \phi_{t, x_{0}} L_{0} d x\right|>\lambda\right\}\right) \\
& \quad \leq \sum_{j=1}^{K} \omega\left(\left\{x_{0}: M\left(\left|f_{j}\right|^{p_{j}}\right)\left(x_{0}\right)>\left(\frac{\varepsilon_{j-1}}{\varepsilon_{j}}\right)^{p_{j}}\right\}\right) \\
& \quad \leq C \sum_{j=1}^{K}\left(\frac{\varepsilon_{j-1}}{\varepsilon_{j}}\right)^{-p_{j}} \int\left|f_{j}\right|^{p_{j}} \omega(x) d x,
\end{aligned}
$$

and the expression minimizes when all the terms that appear in the sum are equal.

Finally, we get the weak type estimate:

$$
\omega\left(\left\{x_{0}: \sup _{t>0}\left|\int \phi_{t, x_{0}} L_{0} d x\right|>\lambda\right\}\right) \leq C \lambda^{-r} \prod_{j=1}^{K}\left\|f_{j}\right\|_{L^{p_{j}}(\omega)^{\prime}}^{r}
$$

and we complete the proof.

\section{The Proof of Theorem 2}

We give the proof for the bilinear operator $T^{-}$only and the proof for $T^{+}$is similar. Let $f \in L^{p}(\omega)$ and $g \in L^{q}(\omega)$. Assume that the parallel Calderón-Zygmund operators $\widetilde{T}_{i}(i=1,2)$ and the parallel bilinear operator $\widetilde{T}^{-}$satisfy (10) and (12). We split $T^{-}(f, g)$ into three parts:

$$
T^{-}(f, g)(x)=T_{1}^{-}(f, g)(x)+T_{2}^{-}(f, g)(x)+\widetilde{T}^{-}(f, g)(x),
$$

where

$$
\begin{aligned}
& T_{1}^{-}(f, g)=\left(T_{1} f-\widetilde{T}_{1} f\right)\left(T_{2} g\right)-\left(T_{2} f-\widetilde{T}_{2} f\right)\left(T_{1} g\right), \\
& T_{2}^{-}(f, g)=\left(\widetilde{T}_{1} f\right)\left(T_{2} g-\widetilde{T}_{2} g\right)-\left(\widetilde{T}_{2} f\right)\left(T_{1} g-\widetilde{T}_{1} g\right) .
\end{aligned}
$$

It follows from Theorem 1 that $\widetilde{T}^{-} \in H^{1}(\omega)$ and

$$
\left\|\widetilde{T}^{-}(f, g)\right\|_{H^{1}(\omega)} \leq C\|f\|_{L^{p}(\omega)}\|g\|_{L^{q}(\omega)} .
$$

Since $H^{1}(\omega) \subset H_{\mathscr{L}}^{1}(\omega)$, we have $\widetilde{T}^{-}(f, g)$ and

$$
\left\|\widetilde{T}^{-}(f, g)\right\|_{H_{\mathcal{I}}^{1}(\omega)} \leq C\|f\|_{L^{p}(\omega)}\|g\|_{L^{q}(\omega)} .
$$

It suffices to show $T_{1}^{-}(f, g)+T_{2}^{-}(f, g) \in H_{\mathscr{L}}^{1}(\omega)$; that is,

$$
\begin{aligned}
\mathscr{T}^{*} & \left(T_{1}^{-}(f, g)+T_{2}^{-}(f, g)\right)(x) \\
& =\sup _{t>0}\left|T_{t}^{\mathscr{L}}\left(T_{1}^{-}(f, g)+T_{2}^{-}(f, g)\right)(x)\right| \in L^{1}(\omega) .
\end{aligned}
$$

For simplicity we write $F(f, g)(x)=T_{1}^{-}(f, g)+T_{2}^{-}(f, g)$. Choose a sequence of points $\left\{x_{(k, \alpha)}\right\}$ and a family of functions $\left\{\psi_{(k, \alpha)}\right\}$ as in Lemma 5. Then

$$
\begin{aligned}
\left\|T_{1}^{-}(f, g)+T_{2}^{-}(f, g)\right\|_{H_{\mathcal{I}}^{1}(\omega)} \\
\leq \sum_{(k, \alpha)}\left(\int_{\left(B_{(k, \alpha)}^{* *}\right)^{c}}+\int_{B_{(k, \alpha)}^{* *}}\right) \\
\quad \times \sup _{t>0}\left|T_{t}^{\mathscr{L}}\left(\psi_{(k, \alpha)} F(f, g)\right)(x)\right| \omega(x) d x \\
=\sum_{(k, \alpha)} I_{(k, \alpha)}+\sum_{(k, \alpha)} J_{(k, \alpha)} .
\end{aligned}
$$

For $I_{(k, \alpha)}$, by using (23), we get

$$
\begin{aligned}
& I_{(k, \alpha)} \\
& =\int_{\left(B_{(k, \alpha)}^{* *}\right)^{c}} \sup _{t>0}\left|\int_{\mathbb{R}^{d}} T_{t}^{\mathscr{L}}(x, y) \psi_{(k, \alpha)}(y) F(f, g)(y) d y\right| \\
& \quad \times \omega(x) d x \\
& \leq C_{N} \int_{\left(B_{(k, \alpha)}^{* *}\right)^{c}} \sup _{t>0} \int_{\mathbb{R}^{d}} \rho(y)^{N} t^{-((d+N) / 2)} e^{-\left(|x-y|^{2} / 5 t\right)} \\
& \times C_{N} \int_{\left(B_{(k, \alpha)}^{* *}\right)^{c}} \int_{\mathbb{R}^{d}} \frac{\rho(x)^{N}}{|x-y|^{d+N}} \\
& \times \mid \psi(k, \alpha) \\
& \quad \times \mid y) F(f, g)(y) \mid d y \omega(x) d x \\
&
\end{aligned}
$$

and for $x \notin B_{k, \alpha}^{* *}$ and $y \in \operatorname{supp} \psi_{(k, \alpha)} \subset B_{(k, \alpha)}^{*}$, Lemma 6 yields $\rho(y) \sim \rho\left(x_{(k, \alpha)}\right)$ and $|x-y| \geq(1 / 2)\left|x-x_{(k, \alpha)}\right|$; then we have

$$
\begin{aligned}
I_{(k, \alpha)} \leq C \int_{B_{(k, \alpha)}^{*}} & \left|\psi_{(k, \alpha)}(y) F(f, g)(y)\right| \\
& \times\left(\int_{\left(B_{(k, \alpha)}^{* *}\right)} \frac{\rho\left(x_{(k, \alpha)}\right)^{N}}{\left|x-x_{(k, \alpha)}\right|^{d+N}} \omega(x) d x\right) d y .
\end{aligned}
$$

For the inner integral we have

$$
\begin{aligned}
\int_{\left(B_{(k, \alpha)}^{* *}\right)} & \frac{\rho\left(x_{(k, \alpha)}\right)^{N}}{\left|x-x_{(k, \alpha)}\right|^{d+N}} \omega(x) d x \\
= & \int_{\left|x-x_{(k, \alpha)}\right|>4 \rho\left(x_{(k, \alpha)}\right)} \frac{\rho\left(x_{(k, \alpha)}\right)^{N}}{\left|x-x_{(k, \alpha)}\right|^{d+N}} \omega(x) d x \\
= & \sum_{j=2}^{\infty} \int_{\left|x-x_{(k, \alpha)}\right| 2^{j} \rho\left(x_{(k, \alpha)}\right)} \frac{\rho\left(x_{(k, \alpha)}\right)^{N}}{\left|x-x_{(k, \alpha)}\right|^{d+N}} \omega(x) d x
\end{aligned}
$$




$$
\begin{aligned}
& \leq \sum_{j=2}^{\infty} \frac{\rho\left(x_{(k, \alpha)}\right)^{N}}{\left(2^{j} \rho\left(x_{(k, \alpha)}\right)\right)^{d+N}} \omega\left(B\left(x_{(k, \alpha)}, 2^{j+1} \rho\left(x_{(k, \alpha)}\right)\right)\right) \\
& \leq C \frac{\omega\left(B_{(k, \alpha)}^{*}\right)}{\left|B_{(k, \alpha)}^{*}\right|} \leq C \omega(y),
\end{aligned}
$$

where $y \in B_{(k, \alpha)}^{*}$. Then we obtain

$$
\begin{aligned}
I_{(k, \alpha)} & \leq C \int_{B_{(k, \alpha)}^{*}}\left|\psi_{(k, \alpha)}(y) F(f, g)(y)\right| \omega(y) d y \\
& \leq C\left\|\psi_{(k, \alpha)} F(f, g)\right\|_{L^{1}(\omega)},
\end{aligned}
$$

and hence

$$
\begin{aligned}
\sum_{(k, \alpha)} I_{(k, \alpha)} & \leq C \sum_{(k, \alpha)}\left\|\psi_{(k, \alpha)} F(f, g)\right\|_{L^{1}(\omega)} \\
& \leq C\|F(f, g)\|_{L^{1}(\omega)} \leq C\|f\|_{L^{p}(\omega)}\|g\|_{L^{q}(\omega)} .
\end{aligned}
$$

To estimate $\sum_{(k, \alpha)} J_{(k, \alpha)}$, we write

$$
\begin{aligned}
& \sum_{(k, \alpha)} J_{(k, \alpha)} \\
& \leq \sum_{(k, \alpha)} \int_{B_{(k, \alpha)}^{* *}} \sup _{t \geq \rho\left(x_{(k, \alpha)}\right)^{2}}\left|T_{t}^{\mathscr{L}}\left(\psi_{(k, \alpha)} F(f, g)\right)(x)\right| \\
& \times \omega(x) d x \\
& \quad+\sum_{(k, \alpha)} \int_{B_{(k, \alpha)}^{* *}} \sup _{0<t<\rho\left(x_{(k, \alpha)}\right)^{2}}\left|T_{t}^{\mathscr{L}}\left(\psi_{(k, \alpha)} F(f, g)\right)(x)\right| \\
& \quad \times \omega(x) d x \\
& =J_{1}+J_{2} . \quad
\end{aligned}
$$

For $J_{1}$, we apply (23) and Lemma 6 to obtain

$$
\begin{array}{r}
\int_{B_{(k, \alpha)}^{* *}} \sup _{t \geq \rho\left(x_{(k, \alpha)}\right)^{2}}\left|T_{t}^{\mathscr{L}}\left(\psi_{(k, \alpha)} F(f, g)\right)(x)\right| \omega(x) d x \\
\leq C_{N} \int_{B_{(k, \alpha)}^{* *}} \sup _{t \geq \rho\left(x_{(k, \alpha)}\right)^{2}} \int_{\mathbb{R}^{d}} \rho(y)^{N} t^{-((d+N) / 2)} e^{-\left(|x-y|^{2} / 5 t\right)} \\
\times\left|\psi_{(k, \alpha)}(y) F(f, g)(y)\right| d y \omega(x) d x \\
\leq C_{N} \int_{B_{(k, \alpha)}^{* *}} \sup _{t \geq \rho\left(x_{(k, \alpha)}\right)^{2}} \int_{\mathbb{R}^{d}} \rho\left(x_{(k, \alpha)}\right)^{N} t^{-((d+N) / 2)} \\
\quad \times\left|\psi_{(k, \alpha)}(y) F(f, g)(y)\right| d y \omega(x) d x
\end{array}
$$

$$
\begin{aligned}
& \leq C_{N} \int_{B_{(k, \alpha)}^{*}}\left|\psi_{(k, \alpha)}(y) F(f, g)(y)\right| \\
& \quad \times\left(\frac{1}{\rho\left(x_{(k, \alpha)}\right)^{d}} \int_{B_{(k, \alpha)}^{* *}} \omega(x) d x\right) d y \\
& \leq C \int_{B_{(k, \alpha)}^{*}}\left|\psi_{(k, \alpha)}(y) F(f, g)(y)\right| \omega(y) d y \\
& \leq C\left\|\psi_{(k, \alpha)} F(f, g)\right\|_{L^{1}(\omega)^{\prime}}
\end{aligned}
$$

and hence

$$
J_{1} \leq C \sum_{(k, \alpha)}\left\|\psi_{(k, \alpha)} F(f, g)\right\|_{L^{1}(\omega)} \leq C\|f\|_{L^{p}(\omega)}\|g\|_{L^{q}(\omega)} .
$$

To estimate $J_{2}$, we denote

$$
\begin{aligned}
& J_{2} \\
& \leq \sum_{(k, \alpha)} \int_{B_{(k, \alpha)}^{* *}} \sup _{0<t<\rho\left(x_{(k, \alpha)}\right)^{2}}\left|\left(T_{t}^{\mathscr{L}}-H_{t}\right)\left(\psi_{(k, \alpha)} F(f, g)\right)(x)\right| \\
& \times \omega(x) d x \\
& +\sum_{(k, \alpha)} \int_{B_{(k, \alpha)}^{* *}} \sup _{0<t<\rho\left(x_{(k, \alpha)}\right)^{2}} \mid H_{t}\left(\psi _ { ( k , \alpha ) } \left(T_{1}^{-}(f, g)\right.\right. \\
& \left.\left.+T_{2}^{-}(f, g)\right)(x)\right) \\
& \times \omega(x) d x \\
& =J_{21}+J_{22} \text {. }
\end{aligned}
$$

For $x \in B_{k, \alpha}^{* *}$ and $y \in \operatorname{supp} \psi_{(k, \alpha)} \subset B_{(k, \alpha)}^{*}$, by Lemma 6 we have $|x-y|<6 \rho\left(x_{(k, \alpha)}\right) \sim \rho(x)$; then the estimate (24) gives

$$
\begin{array}{r}
\int_{B_{(k, \alpha)}^{* *} 0<t<\rho\left(x_{(k, \alpha)}\right)^{2}}\left|\left(T_{t}^{\mathcal{L}}-H_{t}\right)\left(\psi_{(k, \alpha)} F(f, g)\right)(x)\right| \omega(x) d x \\
\leq C \int_{B_{(k, \alpha)}^{* *}} \int_{B_{(k, \alpha)}^{*}} \frac{1}{\rho(x)^{\sigma}|x-y|^{d-\sigma}} \\
\quad \times\left|\psi_{(k, \alpha)}(y) F(f, g)(y)\right| d y \omega(x) d x \\
\leq C \int_{B_{(k, \alpha)}^{*}}\left|\psi_{(k, \alpha)}(y) F(f, g)(y)\right| \\
\quad \times\left(\int_{B_{(k, \alpha)}^{* *}} \frac{\omega(x) d x}{\rho\left(x_{(k, \alpha)}\right)^{\sigma}|x-y|^{d-\sigma}}\right) d y .
\end{array}
$$


For the inner integral, by properties of weight, we have

$$
\begin{aligned}
\int_{B_{(k, \alpha)}^{* *}} & \frac{\omega(x)}{\rho\left(x_{(k, \alpha)}\right)^{\sigma}|x-y|^{d-\sigma}} d x \\
& \leq \int_{|x-y|<8 \rho\left(x_{(k, \alpha)}\right)} \frac{\omega(x)}{\rho\left(x_{(k, \alpha)}\right)^{\sigma}|x-y|^{d-\sigma}} d x \\
& \leq \sum_{j=-2}^{\infty} \int_{|x-y| \sim 2^{-j} \rho\left(x_{(k, \alpha)}\right)} \frac{\omega(x)}{\rho\left(x_{(k, \alpha)}\right)^{\sigma}|x-y|^{d-\sigma}} d x \\
& \leq C \sum_{j=-2}^{\infty} \frac{1}{\left(2^{\sigma}\right)^{j}} \frac{\omega\left(B\left(y, 2^{-j} \rho\left(x_{(k, \alpha)}\right)\right)\right)}{\left(2^{-j} \rho\left(x_{(k, \alpha)}\right)\right)^{d}} \leq C \omega(y),
\end{aligned}
$$

which yield

$$
J_{21} \leq C \sum_{(k, \alpha)}\left\|\psi_{(k, \alpha)} F(f, g)\right\|_{L^{1}(\omega)} \leq C\|f\|_{L^{p}(\omega)}\|g\|_{L^{q}(\omega)} .
$$

Now let us estimate $J_{22}$. For $t>0$, let $\Gamma_{0}=\left\{y \in \mathbb{R}^{d}: \mid x-\right.$ $y \mid<\sqrt{t}\}$ and $\Gamma_{n}=\left\{y \in \mathbb{R}^{d}: 2^{n-1} \sqrt{t} \leq|x-y|<2^{n} \sqrt{t}\right\}, n \in \mathbb{N}$. Then

$$
\begin{aligned}
& H_{t}\left(\psi_{(k, \alpha)} T_{m}^{-}(f, g)\right)(x) \\
& \quad=\sum_{n=0}^{\infty} H_{t}\left(\psi_{(k, \alpha)} T_{m}^{-}(f, g) \chi_{\Gamma_{n}}\right)(x), \quad m=1,2,
\end{aligned}
$$

where $\chi_{\Gamma_{n}}$ denotes the characteristic function of the set $\Gamma_{n}$. Let $\eta(y) \in C_{0}^{\infty}\left(\mathbb{R}^{d}\right)$ with $0 \leq \eta \leq 1$ satisfy $\eta(y)=1$ for $|y|<2$ and $\eta(y)=0$ for $|y|>4$. Set $\eta_{0}^{n}(y)=\eta\left((x-y) / 2^{n} \sqrt{t}\right)$ and $\eta_{1}^{n}(y)=1-\eta_{0}^{n}(y), n \in \mathbb{N} \cup\{0\}$. We split the operator $T_{m}^{-}(f, g)$ into four parts:

$$
\begin{aligned}
T_{m}^{-}(f, g)= & T_{m}^{-}\left(f, \eta_{1}^{n} g\right)+T_{m}^{-}\left(\eta_{1}^{n} f, g\right) \\
& -T_{m}^{-}\left(\eta_{1}^{n} f, \eta_{1}^{n} g\right)+T_{m}^{-}\left(\eta_{0}^{n} f, \eta_{0}^{n} g\right), \quad m=1,2 .
\end{aligned}
$$

We first estimate

$$
\left.\int_{B_{(k, \alpha)}^{* *} 0<t<\rho\left(x_{(k, \alpha)}\right)^{2}} \sup _{t} \mid H_{(k, \alpha)} T_{1}^{-}\left(f, \eta_{1}^{n} g\right) \chi_{\Gamma_{n}}\right)(x) \mid \omega(x) d x
$$

Write

$$
T_{1}^{-}\left(f, \eta_{1}^{n} g\right)=\left(U_{1}^{1} f\right) U_{1}^{2}\left(\eta_{1}^{n} g\right)-\left(U_{2}^{1} f\right) U_{2}^{2}\left(\eta_{1}^{n} g\right)
$$

where

$$
U_{1}^{1}=T_{1}-\widetilde{T}_{1}, \quad U_{2}^{1}=T_{2}-\widetilde{T}_{2}, \quad U_{1}^{2}=T_{2}, \quad U_{2}^{2}=T_{1} .
$$

Then we have

$$
\begin{aligned}
& \left|H_{t}\left(\psi_{(k, \alpha)} T_{1}^{-}\left(f, \eta_{1}^{n} g\right) \chi_{\Gamma_{n}}\right)(x)\right| \\
& \leq \sum_{l=1}^{2} \int_{\Gamma_{n}} H_{t}(x-y)\left|U_{l}^{1} f(y)\right| \\
& \quad \times\left|U_{l}^{2}\left(\eta_{1}^{n} g\right)(y)-U_{l}^{2}\left(\eta_{1}^{n} g\right)(x)\right| d y \\
& \quad+\sum_{l=1}^{2} \int_{\Gamma_{n}} H_{t}(x-y)\left|U_{l}^{1} f(y)\right|\left|U_{l}^{2}\left(\eta_{1}^{n} g\right)(x)\right| d y \\
& \leq C 2^{n d} e^{-2^{2 n-4}} \sum_{l=1}^{2} \frac{1}{\left(2^{n} \sqrt{t}\right)^{d}} \\
& \quad \times \int_{|x-y|<2^{n} \sqrt{t}}\left|U_{l}^{1} f(y)\right| \\
& \quad \times \int_{|x-y|<2^{n} \sqrt{t}}\left|U_{l}^{1} f(y)\right|\left|U_{l}^{2}\left(\eta_{1}^{n} g\right)(x)\right| d y, \\
& \quad+C 2^{n d} e^{-2^{2 n-4}} \sum_{l=1}^{2} \frac{1}{\left(2^{n} \sqrt{t}\right)^{d}} \\
&
\end{aligned}
$$

where we have used (5) in the last inequality. Since $U_{l}^{j}(j, l=$ $1,2)$ are Calderón-Zygmund operators, their kernels satisfy the standard kernel estimate for some $\delta>0$. For $|x-y| \leq$ $2^{n} \sqrt{t}$

$$
\begin{aligned}
& \left|U_{l}^{2}\left(\eta_{1}^{n} g\right)(y)-U_{l}^{2}\left(\eta_{1}^{n} g\right)(x)\right| \\
& \quad \leq \int_{|x-z| \geq 2^{n+1} \sqrt{t}} \frac{|x-y|^{\delta}}{|x-z|^{d+\delta}}\left|\left(\eta_{1}^{n} g\right)(z)\right| d z \leq C M\left(\eta_{1}^{n} g\right)(x),
\end{aligned}
$$

where $M$ is the Hardy-Littlewood maximal operator. Thus,

$$
\begin{aligned}
\left|H_{t}\left(\psi_{(k, \alpha)} T_{1}^{-}\left(f, \eta_{1}^{n} g\right) \chi_{\Gamma_{n}}\right)(x)\right| & \\
\leq C 2^{n d} e^{-2^{2 n-4}} \sum_{l=1}^{2}( & M\left(U_{l}^{1} f\right)(x) M\left(\eta_{1}^{n} g\right)(x) \\
& \left.+M\left(U_{l}^{1} f\right)(x)\left|U_{l}^{2}\left(\eta_{1}^{n} g\right)(x)\right|\right),
\end{aligned}
$$


and hence

$$
\begin{aligned}
& \sum_{(k, \alpha)} \int_{B_{(k, \alpha)}^{* *} 0<t<\rho\left(x_{(k, \alpha)}\right)^{2}} \sup \left|H_{t}\left(\psi_{(k, \alpha)} T_{1}^{-}\left(f, \eta_{1}^{n} g\right) \chi_{\Gamma_{n}}\right)(x)\right| \\
& \quad \times \omega(x) d x \\
& \leq C 2^{n d} e^{-2^{2 n-4}} \int_{\mathbb{R}^{d}} \sum_{l=1}^{2} M\left(U_{l}^{1} f\right)(x) \\
& \times\left(M\left(\eta_{1}^{n} g\right)(x)+\left|U_{l}^{2}\left(\eta_{1}^{n} g\right)(x)\right|\right) \\
& \times C 2^{n d} e^{-2^{2 n-4}} \sum_{l=1}^{2}\left\|M\left(U_{l}^{1} f\right)(x)\right\|_{L^{p}(\omega)} \\
& \times\left(\|M(g)\|_{L^{q}(\omega)}+\left\|U_{l}^{2} g\right\|_{L^{q}(\omega)}\right) \\
& \leq C 2^{n d} e^{-2^{2 n-4}}\|f\|_{L^{p}(\omega)}\|g\|_{L^{q}(\omega)}
\end{aligned}
$$

A similar argument shows that

$$
\begin{gathered}
\sum_{(k, \alpha)} \int_{B_{(k, \alpha)}^{* *} 0<t<\rho\left(x_{(k, \alpha)}\right)^{2}} \sup _{t}\left|H_{t}\left(\psi(k, \alpha) T_{1}^{-}\left(\eta_{1}^{n} f, g\right) \chi_{\Gamma_{n}}\right)(x)\right| \omega(x) d x \\
\leq C 2^{n d} e^{-2^{2 n-4}}\|f\|_{L^{p}(\omega)}\|g\|_{L^{q}(\omega)}, \\
\sum_{(k, \alpha)} \int_{B_{(k, \alpha)}^{* *} 0<t<\rho\left(x_{(k, \alpha)}\right)^{2}} \sup _{t} \mid H_{t}(\psi(k, \alpha) \\
\quad \times \omega(x) d x \\
\left.\quad T_{1}^{-}\left(\eta_{1}^{n} f, \eta_{1}^{n} g\right) \chi_{\Gamma_{n}}\right)(x) \mid \\
\leq C 2^{n d} e^{-2^{2 n-4}}\|f\|_{L^{p}(\omega)}\|g\|_{L^{q}(\omega)} .
\end{gathered}
$$

Finally we consider the term $T_{1}^{-}\left(\eta_{0}^{n} f, \eta_{0}^{n} g\right)$. By using (5) and (10) we have

$$
\begin{gathered}
\sup _{0<t<\rho\left(x_{(k, \alpha)}\right)^{2}}\left|H_{t}\left(\psi_{(k, \alpha)} T_{1}^{-}\left(\eta_{0}^{n} f, \eta_{0}^{n} g\right) \chi_{\Gamma_{n}}\right)(x)\right| \\
\leq \sum_{l=1}^{2} \sup _{0<t<\rho\left(x_{(k, \alpha)}\right)^{2}} \int_{\Gamma_{n}} H_{t}(x-y)\left|U_{l}^{1}\left(\eta_{0}^{n} f\right)(y)\right| \\
\times\left|\psi_{(k, \alpha)}(y)\right|\left|U_{l}^{2}\left(\eta_{0}^{n} g\right)(y)\right| d y \\
\leq \sup _{0<t<\rho\left(x_{(k, \alpha)}\right)^{2}} \int_{\Gamma_{n}} H_{t}(x-y)\left(\int_{\mathbb{R}^{d}}\left|T_{1}(y, z)-\widetilde{T}_{1}(y, z)\right|\right. \\
\times\left|T_{2}\left(\eta_{0}^{n} g\right)(y)\right| d y
\end{gathered}
$$

$$
\begin{aligned}
& +\sup _{0<t<\rho\left(x_{(k, \alpha)}\right)^{2}} \int_{\Gamma_{n}} H_{t}(x-y) \\
& \quad \times\left(\int_{\mathbb{R}^{d}}\left|T_{2}(y, z)-\widetilde{T}_{2}(y, z)\right|\right. \\
& \left.\quad \times\left|\left(\eta_{0}^{n} f\right)(z)\right| d z\right)\left|T_{1}\left(\eta_{0}^{n} g\right)(y)\right| d y \\
& \leq C e^{-2^{2 n-4}} \sup _{0<t<\rho\left(x_{(k, \alpha)}\right)^{2}} t^{-d / 2}\left\|\eta_{0}^{n} g\right\|_{L^{p_{1}\left(\mathbb{R}^{d}\right)}} \\
& \quad \times\left\{\int_{\Gamma_{n}}\left(\int_{\mathbb{R}^{d}} \frac{\left|\left(\eta_{0}^{n} f\right)(z)\right|}{\rho(z)^{\delta}|y-z|^{d-\delta}} d z\right)^{p_{1}^{\prime}} d y\right\}^{1 / p_{1}^{\prime}},
\end{aligned}
$$

where $1 / p_{1}^{\prime}+1 / p_{1}=1$. For $z \in \operatorname{supp} \eta_{0}^{n}=\left\{y \in \mathbb{R}^{d}: \mid x-\right.$ $\left.y \mid \leq 2^{n+2} \sqrt{t}\right\}, x \in B_{(k, \alpha)}^{* *}$, and $s<\rho\left(x_{(k, \alpha)}\right)^{2}$, we have $\mid z-$ $x_{(k, \alpha)} \mid \leq 2^{n+3} \rho\left(x_{(k, \alpha)}\right)$. It follows from Lemma 6 that $\rho(z)^{-\delta} \leq$ $C 2^{n \delta k_{0}} \rho\left(x_{(k, \alpha)}\right)^{-\delta}$ for a fixed constant $k_{0}>0$. Hence, a wellknown result for fractional integrals gives

$$
\begin{aligned}
& \sup _{0<t<\rho\left(x_{(k, \alpha)}\right)^{2}}\left|H_{t}\left(\psi_{(k, \alpha)} T_{1}^{-}\left(\eta_{0}^{n} f, \eta_{0}^{n} g\right) \chi_{\Gamma_{n}}\right)(x)\right| \\
& \leq C 2^{n \delta k_{0}} e^{-2^{2 n-4}} \sup _{0<t<\rho\left(x_{(k, \alpha)}\right)^{2}} t^{-d / 2} \rho\left(x_{(k, \alpha)}\right)^{-\delta} \\
& \times\left\|\left|\eta_{0}^{n} f\right| * \frac{1}{|\cdot|^{d-\delta}}\right\|_{L^{p_{1}^{\prime}\left(\mathbb{R}^{d}\right)}}\left\|\eta_{0}^{n} g\right\|_{L^{p_{1}}\left(\mathbb{R}^{d}\right)} \\
& \leq C 2^{n \delta k_{0}} e^{-2^{2 n-4}} \sup _{0<t<\rho\left(x_{(k, \alpha)}\right)^{2}} \frac{\left(2^{n} \sqrt{t}\right)^{d+\delta}}{\sqrt{t^{d}} \rho\left(x_{(k, \alpha)}\right)^{\delta}} \frac{1}{\left(2^{n} \sqrt{t}\right)^{d+\delta}} \\
& \times\left\|\eta_{0}^{n} f\right\|_{L^{q_{1}}\left(\mathbb{R}^{d}\right)}\left\|\eta_{0}^{n} g\right\|_{L^{p_{1}}\left(\mathbb{R}^{d}\right)} \\
& \leq C 2^{n\left(d+\delta+\delta k_{0}\right)} e^{-2^{2 n-4}} M\left(|f|^{q_{1}}\right)^{1 / q_{1}}(x) M\left(|g|^{p_{1}}\right)^{1 / p_{1}}(x),
\end{aligned}
$$

where $1 / p_{1}^{\prime}=1 / q_{1}-\delta / d$. Since $1 / p_{1}+1 / q_{1}=1+\delta / d>$ $1 / p+1 / q$, we are always able to choose $p_{1}$ and $q_{1}$ such that $1<p_{1}<q$ and $1<q_{1}<p$. Then we get

$$
\begin{aligned}
\sum_{(k, \alpha)} \int_{B_{(k, \alpha)}^{* *}} \sup _{0<t<\rho\left(x_{(k, \alpha)}\right)^{2}}\left|H_{t}\left(\psi_{(k, \alpha)} T_{1}^{-}\left(\eta_{0}^{n} f, \eta_{0}^{n} g\right) \chi_{\Gamma_{n}}\right)(x)\right| \\
\quad \times \omega(x) d x \\
\leq C 2^{n\left(d+\delta+\delta k_{0}\right)} e^{-2^{2 n-4}} \\
\quad \times \int_{\mathbb{R}^{d}} M\left(|f|^{q_{1}}\right)^{1 / q_{1}}(x) M\left(|g|^{p_{1}}\right)^{1 / p_{1}}(x) \omega(x) d x \\
\leq C 2^{n\left(d+\delta+\delta k_{0}\right)} e^{-2^{2 n-4}}
\end{aligned}
$$




$$
\begin{aligned}
& \times\left(\int_{\mathbb{R}^{d}} M\left(|f|^{q_{1}}\right)^{p / q_{1}}(x) \omega(x) d x\right)^{1 / p} \\
\times & \left(\int_{\mathbb{R}^{d}} M\left(|g|^{p_{1}}\right)^{q / p_{1}}(x) \omega(x) d x\right)^{1 / q} \\
\leq & C 2^{n\left(d+\delta+\delta k_{0}\right)} e^{-2^{2 n-4}}\|f\|_{L^{p}(\omega)}\|g\|_{L^{q}(\omega)} .
\end{aligned}
$$

Therefore, we have

$$
\begin{aligned}
& \sum_{(k, \alpha)} \int_{B_{(k, \alpha)}^{* *}} \sup _{0<t<\rho\left(x_{(k, \alpha)}\right)^{2}}\left|H_{t}\left(\psi_{(k, \alpha)} T_{1}^{-}(f, g)\right)(x)\right| \omega(x) d x \\
& \leq \sum_{(k, \alpha)} \int_{B_{(k, \alpha)}^{* *}} \sup _{0<t<\rho\left(x_{(k, \alpha)}\right)^{2}} \sum_{n=0}^{\infty}\left|H_{t}\left(\psi_{(k, \alpha)} T_{1}^{-}(f, g) \chi_{\Gamma_{n}}\right)(x)\right| \\
& \quad \times \omega(x) d x \\
& \leq C\|f\|_{L^{p}(\omega)}\|g\|_{L^{q}(\omega)} \sum_{n=0}^{\infty} 2^{n\left(d+\delta+\delta k_{0}\right)} e^{-2^{2 n-4}} \\
& \leq C\|f\|_{L^{p}(\omega)}\|g\|_{L^{q}(\omega)} .
\end{aligned}
$$

Similarly, we get

$$
\begin{aligned}
& \sum_{(k, \alpha)} \int_{B_{(k, \alpha)}^{* *}} \sup _{0<t<\rho\left(x_{(k, \alpha)}\right)^{2}}\left|H_{t}\left(\psi_{(k, \alpha)} T_{2}^{-}(f, g)\right)(x)\right| \omega(x) d x \\
& \quad \leq C\|f\|_{L^{p}(\omega)}\|g\|_{L^{q}(\omega)} .
\end{aligned}
$$

Then we obtain

$$
J_{22} \leq C\|f\|_{L^{p}(\omega)}\|g\|_{L^{q}(\omega)},
$$

and we complete the proof of Theorem 2 .

\section{Conflict of Interests}

The authors declare that there is no conflict of interests regarding the publication of this paper.

\section{Acknowledgments}

Hua Zhu is supported by Seed Project of Beijing International Studies University. Heping Liu is supported by National Natural Science Foundation of China under Grant no. 11371036 and the Specialized Research Fund for the Doctoral Program of Higher Education of China under Grant no. 2012000110059. Both authors would like to thank the referees for some very valuable suggestions which made this paper more readable.

\section{References}

[1] R. Coifman and L. Grafakos, "Hardy space estimates for multilinear operators," I, Revista Matemática Iberoamericana, vol. 8, pp. 45-67, 1992.
[2] C. Coifman, P. L. Lion, Y. Meyer, and S. Semmes, "Compensated compactness and Hardy spaces," Journal de Mathématiques Pures et Appliquées, vol. 72, pp. 247-286, 1993.

[3] L. Grafakos, "Hardy space estimates for multilinear operators," Revista Matemática Iberoamericana, vol. 8, pp. 69-92, 1992.

[4] C.-C. Lin, Y.-C. Lin, H. Liu, and Y. Liu, "Bilinear operators associated with Schrödinger operators," Studia Mathematica, vol. 205, no. 3, pp. 281-295, 2011.

[5] J. Garca-Cuerva and K. S. Kazarian, "Calderón-Zygmund operators and unconditional bases of weighted Hardy spaces," Studia Mathematica, vol. 109, no. 3, pp. 255-276, 1994.

[6] J. Stromberg and A. Torchinsky, Weighted Hardy Spaces, Lecture Notes in Math, Springer, Berlin, Germany, 1989.

[7] T. Quek and D. Yang, "Calderón-zygmund-type operators on weighted weak hardy spaces over $\mathbb{R}^{n}$," Acta Mathematica Sinica, English Series, vol. 16, no. 1, pp. 141-160, 2000.

[8] R. Feerman and F. Soria, "The space weak $\mathrm{H}^{1}$," Studia Mathematica, vol. 85, pp. 1-16, 1987.

[9] H. Liu, The Weak $H^{p}$ Spaces on Homogeneous Groups, Lecture Notes in Math, Springer, Berlin, Germany, 1991.

[10] J. Alvarez, " $H^{p}$ and weak $H^{p}$ continuity of calderón-zygmund type operators, fourier analysis: analytic and geometric aspects," Marcel Dekker, pp. 17-34, 1994.

[11] F. W. Gehring, "The $\mathrm{L}^{p}$-integrability of the partial derivatives of A quasiconformal mapping," Acta Mathematica, vol. 130, no. 1, pp. 265-277, 1973.

[12] J. A. Goldstein, Semigroups of Linear Operators and Applications, Oxford University Press, New York, NY, USA, 1985.

[13] H. Liu, L. Tang, and H. Zhu, "Weighted Hardy spaces and BMO spaces associated with Schrödinger operators," Mathematische Nachrichten, vol. 285, no. 17-18, pp. 2173-2207, 2012.

[14] Z. Shen, " $\mathrm{L}^{p}$ estimates for Schrödinger operators with certain potentials," Annales de l'institut Fourier, vol. 45, no. 2, pp. 513546, 1995.

[15] J. Dziubański and J. Zienkiewicz, "Hardy space $\mathrm{H}^{1}$ associated to Schrödinger operator with potential satisfying reverse holder inequality," Revista Matematica Iberoamericana, vol. 15, no. 2, pp. 279-296, 1999.

[16] J. Dziubański, G. Garrigós, T. Martínez, J. L. Torrea, and J. Zienkiewicz, "BMO spaces related to Schrödinger operators with potentials satisfying a reverse Hölder inequality," Mathematische Zeitschrift, vol. 249, no. 2, pp. 329-356, 2005.

[17] J. Duoandikoetxea, Fourier Analysis, Graduate Studies in Mathematics, vol. 29, American Mathematical Society, Providence, RI, USA, 2000.

[18] L. Grafakos, Classical and Modern Fourier Analysis, Pearson Edu, New Jersey, NJ, USA, 2004.

[19] E. M. Stein, Harmonic Analysis: Real Variable Methods, Orthogonality, and Oscillatory Integrals, Princeton University Press, Princeton, NJ, USA, 1993. 


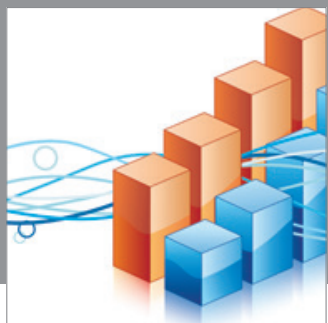

Advances in

Operations Research

mansans

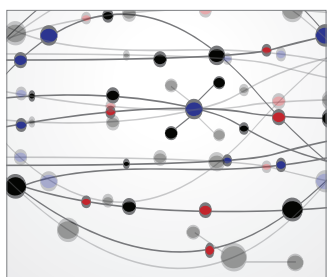

The Scientific World Journal
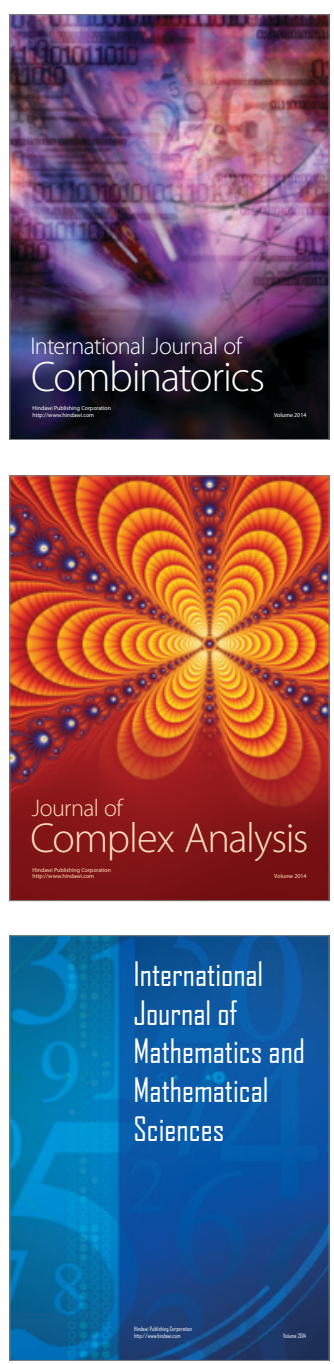
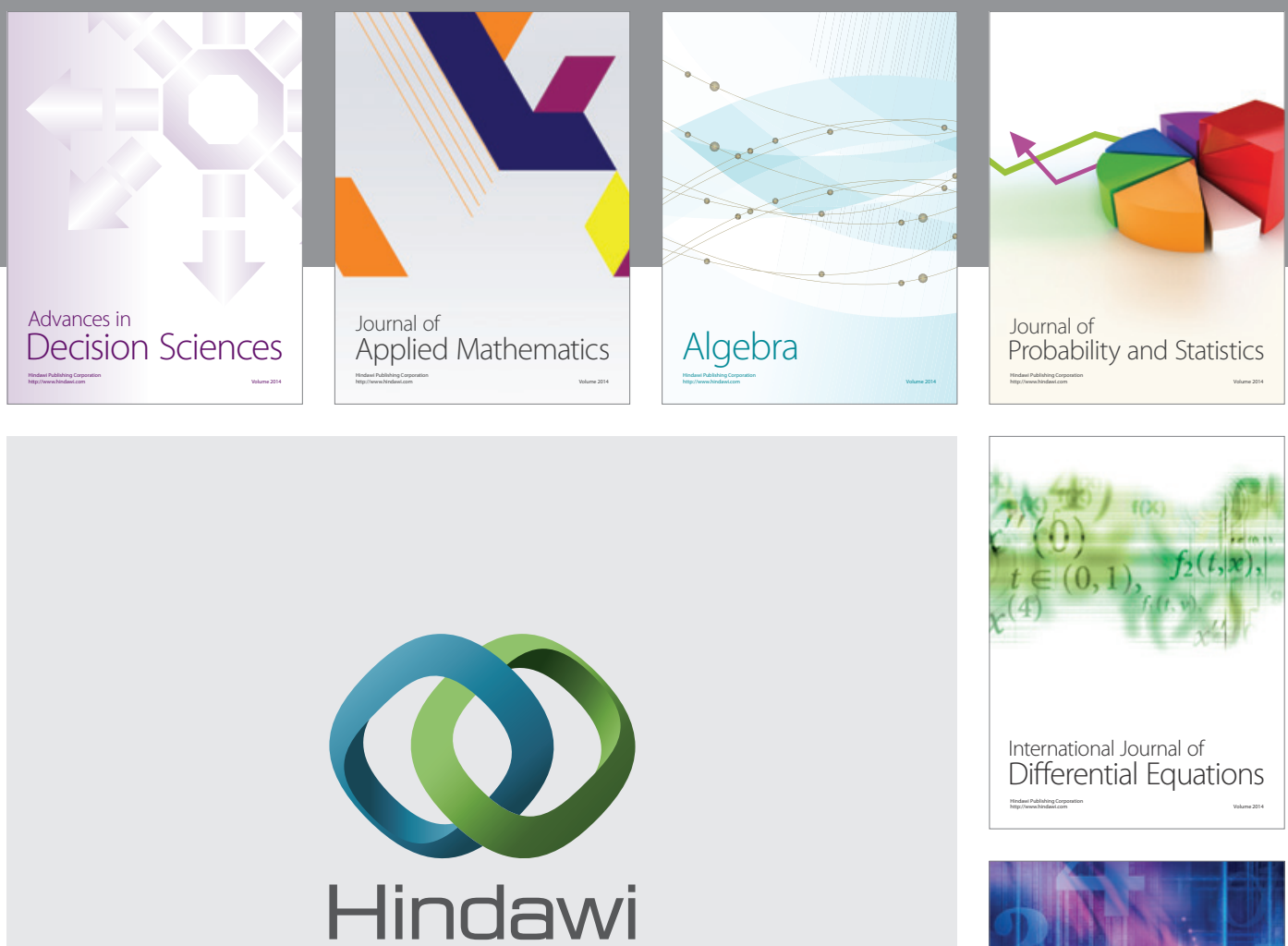

Submit your manuscripts at http://www.hindawi.com
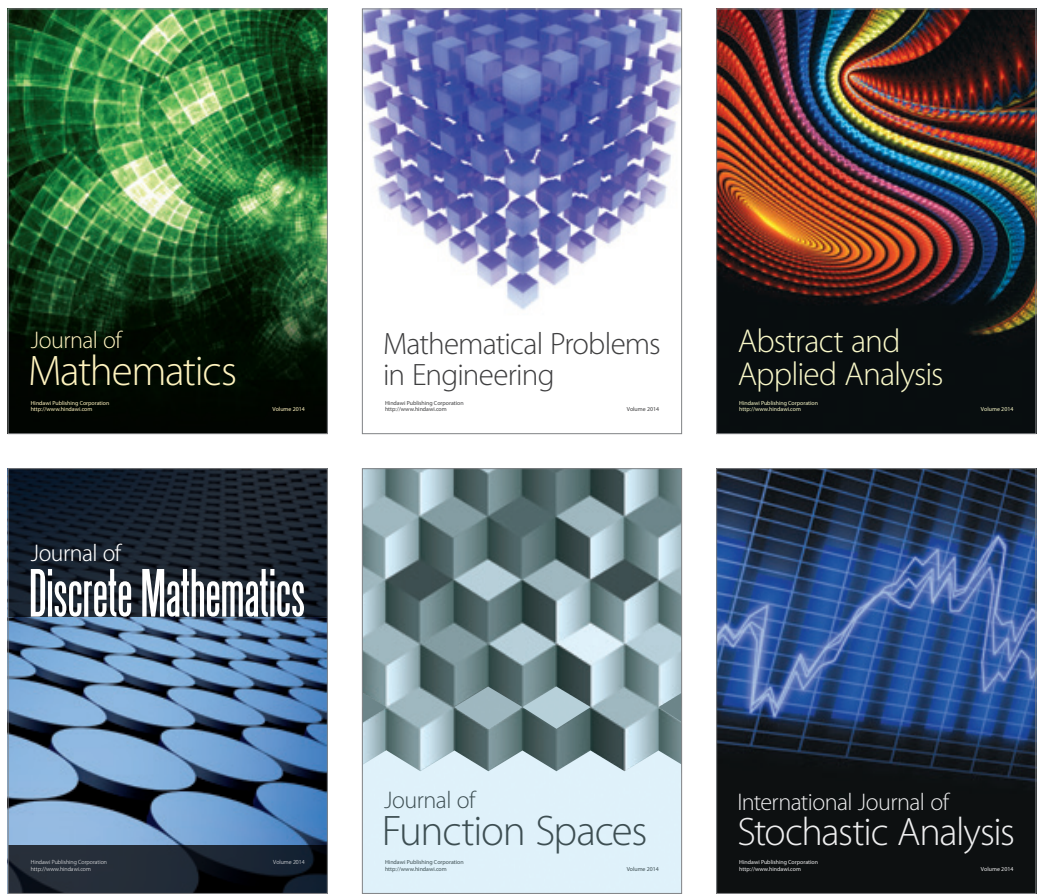

Journal of

Function Spaces

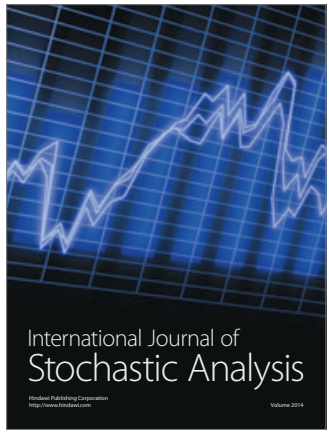

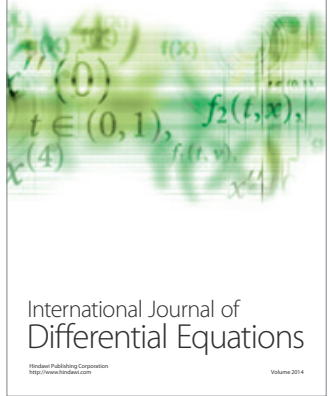
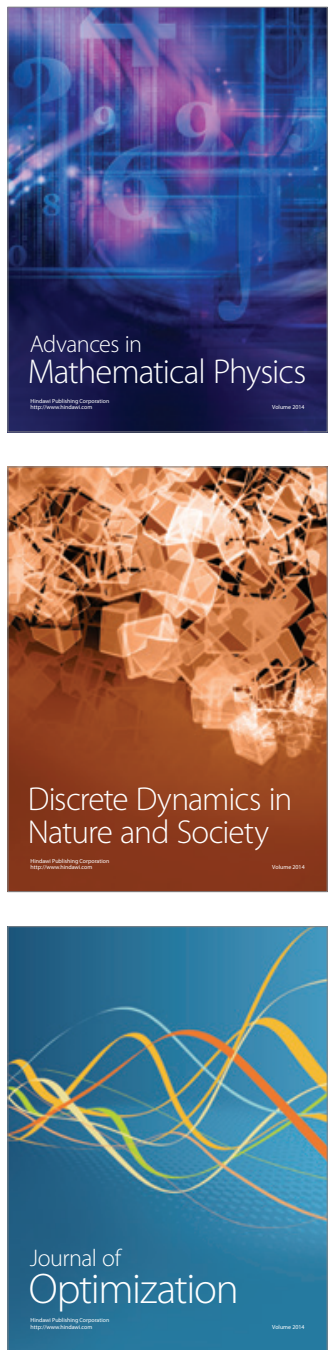